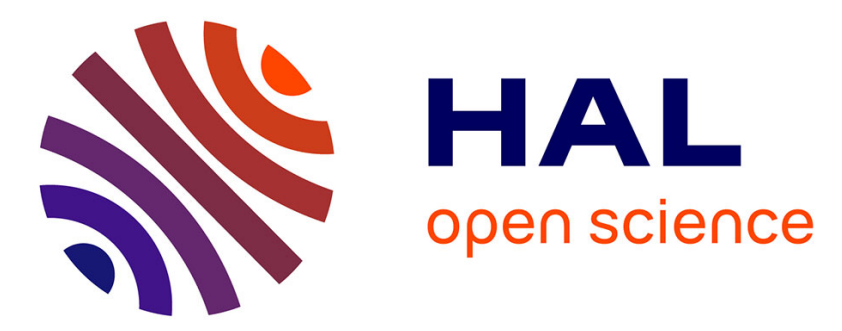

\title{
Comparison of the laser performance of various neodymium doped materials in a compact diode pumped cavity
}

\author{
C. Borel, N. Herlet, R. Templier, C. Calvat, C. Wyon
}

\section{- To cite this version:}

C. Borel, N. Herlet, R. Templier, C. Calvat, C. Wyon. Comparison of the laser performance of various neodymium doped materials in a compact diode pumped cavity. Journal de Physique IV Proceedings, 1994, 04 (C4), pp.C4-549-C4-552. 10.1051/jp4:19944132 . jpa-00252584

HAL Id: jpa-00252584

https://hal.science/jpa-00252584

Submitted on 1 Jan 1994

HAL is a multi-disciplinary open access archive for the deposit and dissemination of scientific research documents, whether they are published or not. The documents may come from teaching and research institutions in France or abroad, or from public or private research centers.
L'archive ouverte pluridisciplinaire HAL, est destinée au dépôt et à la diffusion de documents scientifiques de niveau recherche, publiés ou non, émanant des établissements d'enseignement et de recherche français ou étrangers, des laboratoires publics ou privés. 


\title{
Comparison of the laser performance of various neodymium doped materials in a compact diode pumped cavity
}

\author{
C. BOREL, N. HERLET, R. TEMPLIER, C. CALVAT and C. WYON \\ LETI, CEA-Technologie Avancées, DOPT-SMDO, CENG 85X, 38041 Grenoble cedex, France
}

\section{INTRODUCTION}

The need for compact and highly efficient laser sources has led us to study new neodymium doped materials, better matched to diode pumping than the commercially available Nd:YAG and Nd:YLF. Typical Nd doped single crystals with highly ordered structure exhibit narrow absorption bands (such as YAG). Then, precise control of the diode wavelength around $800 \mathrm{~nm}$ (by the diode temperature adjustment) is required to efficiently pump such crystals but is detrimental to the wall plug efficiency.

On the opposite, disordered crystal structures usually present broadening of the absorption bands characteristic of the lanthanide ions, but are often correlated with a decrease in the stimulated emission cross section, and thus in the laser performance. Yttrium vanadate $\mathrm{YVO}_{4}$ is the only well known lattice which simultaneously presents a broad absorption band around $800 \mathrm{~nm}$ and a narrow and intense emission band in the near infra-red range. Nevertheless, its relatively short lifetime (about $90 \mu \mathrm{s}$ ) doesn't allow an efficient energy storage for pulsed applications, and its crystal growth is especially tough. Another point of interest could be a large absorption coefficient well fitted to microchip lasers application.

Crystals with various structure have been grown by the Czochralski method, spectroscopically characterized and tested in a diode pumped cavity.

\section{CRYSTAL GROWTH}

The structure, melting point and growth conditions of the different Nd doped laser crystals are presented on Table I.

\section{OPTICAL PROPERTIES}

Polarized absorption and emission spectra of the different $\mathrm{Nd}$ doped materials have been recorded at room temperature and are used to calculate the polarized absorption cross sections near $800 \mathrm{~nm}$, zone of interest for the AlAsGa diode pumping. The absorption cross section of $\mathrm{Nd}_{\mathrm{O}} \mathrm{Y}_{2} \mathrm{SiO}_{5}$ is compared to the one of $\mathrm{Nd}$ :YAG on Figure 1.

Using the Judd-Ofelt theory [1,2], the adjustable phenomelogical Judd-Ofelt parameters $\Omega_{t}$ have been calculated from the measured oscillator strengths (table II). These Judd-Ofelt parameters have then be used to determine the electric dipolar transition line strengths $\mathrm{f}^{\mathrm{DE}} \mathrm{JJ}^{\prime}$ between any two levels of $\mathrm{Nd}^{3+}$ in crystals, and especially the radiative emission rates $\mathrm{A}^{\mathrm{DE}} \mathrm{JJ}^{\prime}$, branching ratio $\beta$ and the radiative lifetime $\tau_{\mathrm{r}}$.

According to the theory used by FUCHTBAUER-LADENBURG [3], the absolute polarized emission cross section have been calculated (Table II) by knowing the branching ratio and the radiative lifetime: 


$$
\sigma^{p}(\lambda)=\frac{3 . \lambda^{2} \cdot \beta}{8 \cdot \pi \cdot n^{2} \cdot \tau_{r} c} \sum_{p} \int_{\frac{I^{p}(\lambda) \cdot d \lambda}{\lambda^{2}}}
$$

where $\operatorname{IP}(\lambda)$ is the relative fluorescence intensity

$\beta$ and $\tau_{\mathrm{r}}$ are respectively the branching ratio and the radiative lifetime previously calculated by the Judd-Ofelt theory.

\section{LASER TESTS IN A DIODE PUMPING CAVITY}

Uncoated rods $5 \mathrm{~mm}$ long and $5 \mathrm{~mm}$ in diameter from different crystals have been tested in a cavity longitudinally pumped by a 1W SDL 2460 laser diode. The cavity was plano-concave with an output mirror radius $R o c=10 \mathrm{~cm}$. The waist of the laser cavity was $\omega c=130 \mu \mathrm{m}$. Table III and Figure 2 present the laser performance of the materials tested for different output couplers transmissions.

The dependence of the laser output power versus the diode temperature has also been measured for YSO, YAG and YVO4. The amplitude of the variation $\Delta \rho 0^{\prime} \rho 0^{m a x}$, where $\rho 0$ is the optical efficiency $\left(P_{\text {out }} / P_{\text {inc }}\right.$ ) has then been calculated for an arbitrary chosen $20^{\circ} \mathrm{C}$ variation in the diode temperature. For comparison, the Full Width at Half Maximum (FWHM) of the absorption coefficient has been measured from the absorption spectra and are reported on the following table:

\begin{tabular}{|c|c|c|c|}
\hline MATERIAL & YSO (pol b) & YAG & YVO $_{4}$ \\
\hline$\Delta \rho 0^{\prime} \rho 0^{\max }$ & $29 \%$ & $64 \%$ & $\sim 7 \%$ \\
\hline FWHM $(\mathrm{nm})$ & 2 & 1,5 & 9 \\
\hline
\end{tabular}

\section{CONCLUSION}

As predicted, $\mathrm{Nd}: \mathrm{YVO}_{4}$ presents the best laser performance as a diode pumped material (high slope efficiency nearly independent of the diode temperature). However its tough crystal growth still limits its application.

Among the other Nd doped single crystals studied here, Nd:YSO seems to be one of the most promising laser materials:

- It reaches slope efficiencies of $54 \%$ for uncoated rods, which is slightly superior to the Nd:YAG performance without adjustment of any parameters.

- Crystals of large sizes and good optical quality can be grown, especially due to the large distribution coefficient of the neodymium ions in the lattice $(k \sim 0,7)$.

- Its large absorption cross section makes it an interesting material for compact laser designs.

- Thanks to its absorption band near $810 \mathrm{~nm}$, slightly broader than the YAG one, it could be one of the possible trade-off between increased inhomogenous line broadening and reduced emission cross section and slope efficiency.

\section{REFERENCES}

[1] - B. R. JUDD, Phys. Rev., vol 127, p 750, (1962)

[2] - G. S. OFELT, J. of Chem. Phys., vol 37, p 511, (1962)

[3] - W. F. KRUPKE, M. D. SHINN, J. E. MARRION, J. A. CAIRD and S.

E. STOKOWSKI, J. Opt. Soc. Amer., B3, p 102, (1986) 


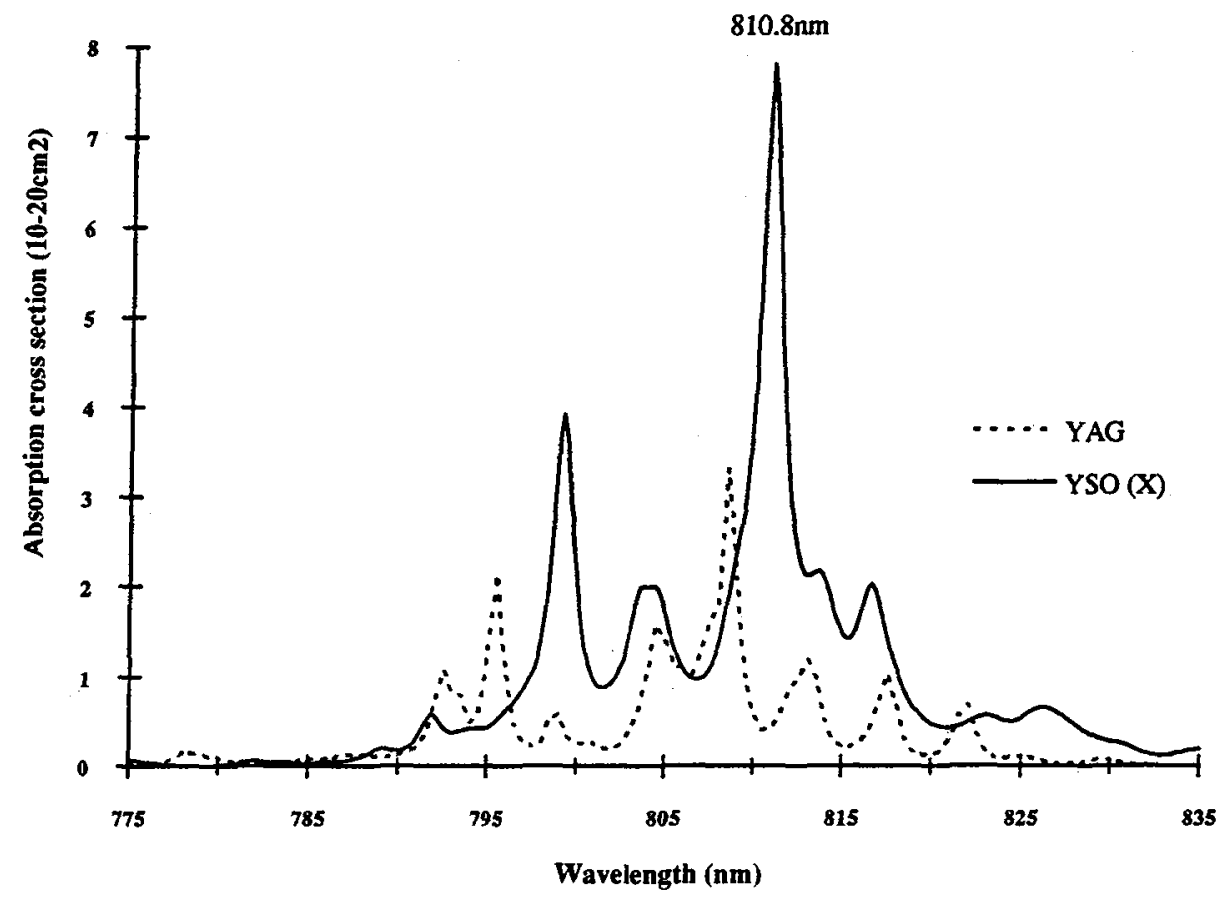

Figure 1: Absorption band of Nd: YSO (X-axis)

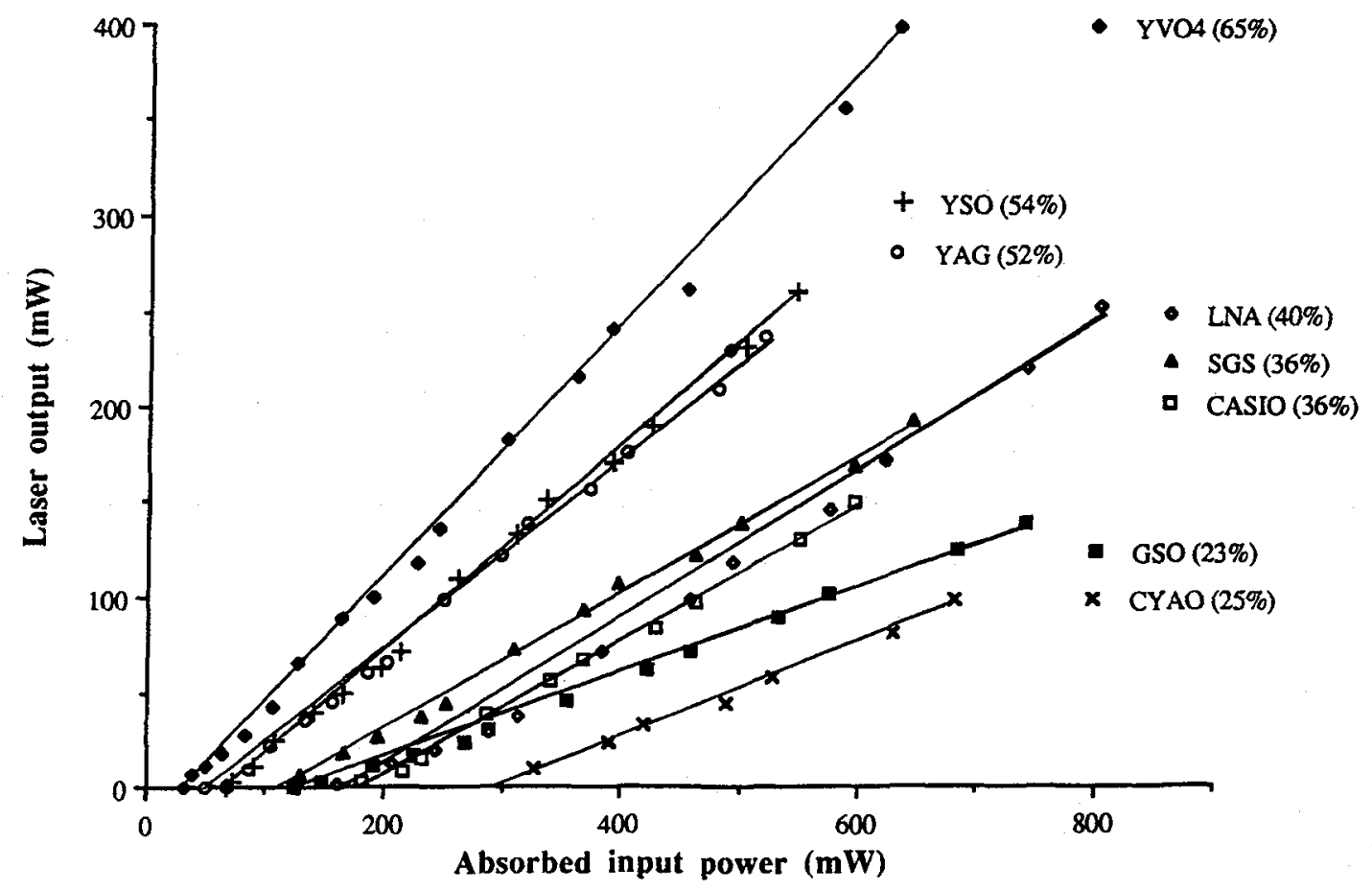

Figure 2: Laser Efficiency of different $\mathrm{Nd}$ doped laser Materials 


\begin{tabular}{|c|c|c|c|c|c|c|}
\hline MATERIAL & Structure & $\begin{array}{l}\text { Melting point } \\
\left({ }^{\circ} \mathrm{C}\right)\end{array}$ & $\begin{array}{c}\mathrm{Nd}^{3+} \\
\text { concentration } \\
\text { in the melt } \\
(\%)\end{array}$ & \begin{tabular}{|c|}
$\mathrm{Nd}^{3+}$ \\
concentration \\
in the melt \\
$\left(10^{20}\right.$ at $\left./ \mathrm{cm}^{3}\right)$
\end{tabular} & $\begin{array}{c}\text { Growth } \\
\text { direction and } \\
\text { atmosphere }\end{array}$ & $\begin{array}{c}\text { Distribution } \\
\text { coefficient } \\
\text { (k) }\end{array}$ \\
\hline $\begin{array}{c}\text { YAG } \\
\mathrm{Y}_{3} \mathrm{Al}_{5} \mathrm{O}_{12}\end{array}$ & $\begin{array}{l}\text { Gamet } \\
\text { (Cubic) }\end{array}$ & -1950 & 1,1 & 1,52 & $\begin{array}{l}\text { sample from } \\
\text { Crismatec }\end{array}$ & $\mathrm{k} \sim 0,18$ \\
\hline $\mathrm{YVO}_{4}$ & Tetragonal & $\sim 1850$ & 2 & 2 & $\begin{array}{l}\text { sample from } \\
\text { NEC }\end{array}$ & \\
\hline $\begin{array}{c}\text { YSO } \\
\mathrm{Y}_{2} \mathrm{SiO}_{5} \\
\end{array}$ & Monoclinic & 1930 & 1 & 1,87 & $\begin{array}{c}<010>=b \\
N_{2}\end{array}$ & $\mathbf{k} \sim 0,7$ \\
\hline $\begin{array}{c}\text { GSO } \\
\mathrm{Gd}_{2} \mathrm{SiO}_{5}\end{array}$ & Monoclinic & $\sim 1900$ & 2 & 3,86 & $\begin{array}{c}<104> \\
\mathrm{N}_{2}\end{array}$ & $\mathrm{k}=1$ \\
\hline $\begin{array}{c}\mathrm{SGS} \\
\mathrm{Sr}_{2} \mathrm{Gd}_{8}\left(\mathrm{SiO}_{4}\right)_{6} \mathrm{O}_{2}\end{array}$ & $\begin{array}{c}\text { Apatite } \\
\text { (hexagonal) }\end{array}$ & $\sim 1900$ & 1,5 & 2,22 & $\begin{array}{c}\left\langle 00^{21}>\right. \\
\mathrm{N}_{2}\end{array}$ & $\mathrm{k}=1$ \\
\hline $\begin{array}{c}\text { CASIO } \\
\mathrm{Ca}_{2} \mathrm{Al}_{2} \mathrm{SiO}_{7} \\
\end{array}$ & $\begin{array}{c}\text { Gehlenite } \\
\text { (tetragonal) }\end{array}$ & 1580 & 1 & 1,34 & $\begin{array}{c}<110> \\
\mathrm{N}_{2}\end{array}$ & $\mathrm{k}>1$ \\
\hline $\begin{array}{c}\text { LMA } \\
\text { LaMgAl }_{11} \mathrm{O}_{19} \\
\end{array}$ & hexagonal & 1910 & 13 & 4,42 & $\begin{array}{l}<110>\text { or } \\
<100>, N_{2}\end{array}$ & $\mathrm{k}=0,7-0,8$ \\
\hline $\begin{array}{c}\text { CYAO } \\
\text { CaYAlO }_{4}\end{array}$ & tetragonal & 1810 & I & $\overline{1,3}$ & $\begin{array}{c}<100> \\
\mathrm{N}_{2}\end{array}$ & $k=1$ \\
\hline
\end{tabular}

Table I: Presentation of the different Nd doped crystals

\begin{tabular}{|c|c|c|c|c|c|c|c|c|}
\hline Material & $\begin{array}{c}\Omega_{2} \\
\left(10-20 \mathrm{~cm}^{2}\right) \\
\end{array}$ & $\begin{array}{c}\Omega_{4} \\
\left(10-20 \mathrm{~cm}^{2}\right)\end{array}$ & $\begin{array}{c}\Omega_{6} \\
(10-20 \\
\mathrm{cm}\end{array}$ & $X=\Omega 4 / \Omega 6$ & $\beta$ & $\begin{array}{c}\tau_{r} \\
(\mu s)\end{array}$ & $\begin{array}{c}\sigma_{\mathrm{e}} \\
\left(10^{\left.-19^{2} \mathrm{~cm}^{2}\right)}\right.\end{array}$ & $\begin{array}{c}\sigma_{e} \cdot \tau_{r} \\
\left(10^{-25}\right)\end{array}$ \\
\hline $\mathrm{YAG}$ & 0,16 & 3,2 & 4,76 & 0,67 & 0,5 & 258 & 2,25 & 581 \\
\hline $\mathrm{YVO}_{4}$ & - & - & - & - & - & $98^{*}$ & $10-20$ & $980-1960$ \\
\hline $\mathrm{GSO}$ & 0,65 & 3,53 & 4,86 & 0,73 & 0,48 & 230 & 0,96 & 221 \\
\hline YSO & 2,84 & 4,78 & 5,41 & 0,88 & 0,46 & 220 & 0,87 & 191 \\
\hline $\mathrm{CaYAlO}_{4}$ & 1,59 & 7,93 & 7,90 & 1 & 0,45 & 140 & 0,77 & 108 \\
\hline SGS & 3,36 & 5,78 & 5,83 & 0,99 & 0,46 & 199 & 0,65 & 129 \\
\hline LMA & 1,23 & 1,75 & 2,24 & 0,78 & 0,47 & 560 & 0,58 & 325 \\
\hline CASIO & 1,09 & 3,6 & 4,84 & 0,74 & 0,48 & 275 & 0,5 & 138 \\
\hline
\end{tabular}

\section{*: Measured fluorescence lifetime}

Table II: Judd Ofelt Parameters

\begin{tabular}{|c|c|c|c|c|c|c|c|c|c|c|}
\hline Material & $\begin{array}{l}\text { CNd3+ } \\
\text { in the } \\
\text { melt } \\
(E 20 \\
\text { at/cm3) }\end{array}$ & $\begin{array}{l}\text { Absorption } \\
\text { coeff. at } \\
\lambda_{\text {pump, in }} \\
\text { the cavity } \\
\left(\mathrm{cm}^{-1}\right)\end{array}$ & $\begin{array}{c}\text { absorption } \\
\text { coeff. at } \\
\lambda_{\text {abs }} \max \\
\left(\mathrm{cm}^{-1}\right)\end{array}$ & $\begin{array}{c}\text { FWHM } \\
\text { in } \\
\text { absorp- } \\
\text { tion } \\
(n m)\end{array}$ & $\begin{array}{c}\text { Fluores- } \\
\text { cence } \\
\text { lifetime } \\
(\mu s)\end{array}$ & $\begin{array}{l}\text { rod axis } \\
(l=5 \mathrm{~mm})\end{array}$ & $\begin{array}{c}\text { Pth abs } \\
(m W)\end{array}$ & $\begin{array}{l}\text { Pout } \\
\max \\
(m W)\end{array}$ & 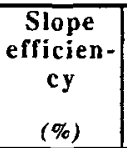 & $\begin{array}{c}\text { Output } \\
\text { mirror } \\
\text { reflec- } \\
\text { tivity } \\
(\%)\end{array}$ \\
\hline$\overline{\mathbf{Y A G}}$ & 1,52 & $\begin{array}{c}3,41 \\
\text { at } 806 \mathrm{~nm}\end{array}$ & $\begin{array}{c}11,3 \\
\text { at } 808,5 \mathrm{~nm}\end{array}$ & 1,5 & 255 & a & $\pi: 49$ & 235,8 & 52 & 94,8 \\
\hline $\mathrm{YVO}_{4}$ & 2 & $\begin{array}{c}11,5 \\
\text { at } 809,4 \mathrm{~nm} \\
\end{array}$ & & $\overline{9}$ & & $\stackrel{a}{(4 \mathrm{~mm})}$ & $\pi: 32$ & 396,8 & 65 & 92 \\
\hline$\overline{L N A}$ & 4,42 & $\begin{array}{c}\alpha: 4,54 \\
\text { at } 798 \mathrm{~nm}\end{array}$ & & 17,3 & 260 & $c$ & $\alpha: 168$ & 251,1 & 40 & 97,25 \\
\hline \multirow[t]{2}{*}{$\overline{\text { SGS }}$} & \multirow[t]{2}{*}{$\begin{array}{c}2,22 \\
(\mathrm{k}=1)\end{array}$} & $\begin{array}{c}\sigma: 6,6 \\
\text { at } 808,5 \mathrm{~nm}\end{array}$ & $\begin{array}{c}9,6 \\
\text { at } 809,5 \mathrm{~nm}\end{array}$ & $\overline{4,1}$ & \multirow[t]{2}{*}{168} & \multirow[t]{2}{*}{ a } & $\sigma: 138$ & 169,3 & 34 & 97,25 \\
\hline & & $\begin{array}{c}\pi: 8,5 \\
\text { at } 808,5 \mathrm{~nm} \\
\end{array}$ & $\begin{array}{c}15,3 \\
\text { at } 809,5 \mathrm{~nm} \\
\end{array}$ & 3,8 & & & $\pi: 134$ & 191,5 & 36 & $97, \overline{25}$ \\
\hline \multirow{3}{*}{$\begin{array}{l}\text { GSO } \\
b=X \\
P 1=Y \\
P 2=Z\end{array}$} & \multirow[t]{3}{*}{3,86} & $\mathrm{E} / / \mathrm{X}$ & $\begin{array}{c}17,4 \\
\text { at } 804,5 \mathrm{~nm} \\
\end{array}$ & 2 & \multirow{3}{*}{185} & \multirow{3}{*}{ b } & & & & \\
\hline & & $\begin{array}{l}\mathrm{E} / \mathrm{Y}: 7,4 \\
\text { at } 799 \mathrm{~nm}\end{array}$ & $\begin{array}{c}14,1 \\
\text { at } 801 \mathrm{~nm}\end{array}$ & 2,3 & & & $\mathrm{E} / / \mathrm{Y}: 134$ & 138,8 & 23 & 96,9 \\
\hline & & $\begin{array}{l}\mathrm{E} / / \mathrm{Z}: 7,8 \\
\text { at } 800 \mathrm{~nm}\end{array}$ & $\begin{array}{c}17,4 \\
\text { at } 810 \mathrm{~nm} \\
\end{array}$ & 1,6 & & & $\mathrm{E} / / \mathrm{Z}: 198$ & 122,8 & 22 & 96,9 \\
\hline $\begin{array}{l}\mathrm{YSO} \\
\mathrm{b}=\mathrm{X}\end{array}$ & \multirow{2}{*}{$(k=0,6)$} & $\begin{array}{l}\mathrm{E} / \mathrm{X}: 5,4 \\
\text { at } 810 \mathrm{~nm}\end{array}$ & $\begin{array}{c}10,2 \\
\text { at } 810,8 \mathrm{~nm}\end{array}$ & 1,8 & \multirow[t]{2}{*}{$\sim 200$} & \multirow[t]{2}{*}{ D1 } & $\mathrm{E} / / \mathrm{b}: 69$ & 259,5 & $\overline{54}$ & 97,25 \\
\hline $\begin{array}{l}\mathrm{D} 1=\mathrm{Y} \\
\mathrm{D} 2=\mathrm{Z} \\
\end{array}$ & & $\begin{array}{l}\mathrm{E} / / \mathrm{Z}: 3,9 \\
\text { at } 810 \mathrm{~nm}\end{array}$ & $\begin{array}{c}6,7 \\
\text { at } 810,8 \mathrm{~nm} \\
\end{array}$ & 1,8 & & & $\mathrm{E} / / \mathrm{D} 2: 80$ & 200 & $\overline{48}$ & 97,25 \\
\hline CASIO & 1,3 & $\begin{array}{c}\alpha: 7,10 \\
\text { at } 807 \mathrm{~nm}\end{array}$ & & 4,9 & 276 & $\mathrm{c}$ & $\alpha: 198$ & 149,9 & $\overline{36}$ & 95,9 \\
\hline CYAO & $\overline{1,3}$ & $\begin{array}{c}\alpha: 9 \\
\text { at } 806 \mathrm{~nm}\end{array}$ & $\begin{array}{c}9,52 \text { at } \\
806,5 \mathrm{~nm}\end{array}$ & 4,3 & 123 & c & $\alpha: 317$ & 98,8 & 25 & 94,8 \\
\hline
\end{tabular}

